\title{
Investigation on Preparation and Porosity of YSZ/PTFE Microporous Membrane Modified by PTFE Resin Emulsion
}

\author{
Jinli Du \\ School of Materials Science and Engineering, Tianjin Polytechnic University \\ No. 63 Chenglin Road, Hedong District, Tianjin 300160, China \\ E-mail: dj1918918@126.com \\ Xinhua Deng (Corresponding author) \\ School of Materials Science and Engineering, Tianjin Polytechnic University \\ No. 63 Chenglin Road, Hedong District, Tianjin 300160, China \\ E-mail: dengxinhua0829@163.com
}

Yuan Sun

School of Materials Science and Engineering, Tianjin Polytechnic University

No. 63 Chenglin Road, Hedong District, Tianjin 300160, China

\begin{abstract}
In this paper, YSZ/PTFE composite microporous membrane was prepared by mechanical tensile method, using PTFE resin emulsion as material and YSZ micro/nanoparticles as reinforcer. The performance of YSZ/PTFE composite microporous membrane was characterized by mechanical property testing. The effects of the dispersant PVA, the draw ratio, YSZ content and calcination temperature on the porosity of composite microporous membrane were systematically investigated. The results showed that the porosity increased significantly as PVA added; with the increase of YSZ content, the porosity gradually increased, but the YSZ content was not over $20 \%$; the porosity reached $73.09 \%$ when the draw ratio 3.5 times, YSZ content $8 \%$, and the calcination temperature $320^{\circ} \mathrm{C}$.
\end{abstract}

Keywords: PTFE, YSZ, Microporous membrane, Porosity

\section{Introduction}

Polytetrafluoroethylene (PTFE) has many excellent characteristics such as low permittivity, (Xie, 2007, pp. 32-39 and $\mathrm{Xi}, 2008$, pp. 7469-7476), high water repellency, high chemical resistance and high heat resistance.( Yuan, 2005, pp. 7-11, Fang, 2001, pp. 13-16, Liu, 2004, pp.340-346, Murali, 2009, pp. 290-295, Kim, 2008 , pp. 654-657 and Wang, 2004, pp. 26-28). PTFE membrane is a new membrane material, has become one of indispensable material in the cutting edge of science and modern industry. There are many scholars have studied of microporous membrane, (Wei, 2005, pp. 27-30, Huai, 2010 and Chen, 2003, pp. 20-22), but few people use PTFE resin emulsion as raw material. Recently, imperfect porosity of pure PTFE membrane has greatly limited its scope of application. Thus a lot of efforts have been continuously made to improve the porosity of PTFE membrane by means of inorganic or organic compound inclusion.

Domestic and foreign researchers through the filled methods improve PTFE membrane's strength, hydrophilicity, abrasion resistance, pressure resistance, or give a special performance to PTFE membrane, such as antibacterial activity. (Sun, 2009, pp. 75-77 and Bao, 2006, pp. 8-11).

Yttria-stabilized zirconia (YSZ) is the most commonly used filler in recent years. YSZ is an important functional oxide for many structural and electronic applications, such as solid oxide fuel cells (Bao, 2007), gas sensor (Liu, 2004, pp. 340-346) and automotive exhaust three-way catalysts (Murali, 2009, pp. 290-295) because of its excellent chemical resistance, refractory character, oxygen ionic conductivity and olymorphous nature. In view of these applications, controlling the porosity of these systems is highly desirable (Hung, 2006, pp. 225-230). So the samples were made by adding micro/nanometer YSZ into PTFE for improving the porosity.

In this article, the purpose of this work is to study the porosity of the PTFE composite microporous membrane filled with YSZ. Some insights into the porosity of YSZ/PTFE composite microporous membrane are also given. 
This study may be helpful for investigating potential applications of the composite microporous membrane.

\section{Experiments}

\subsection{Materials}

PTFE resin emulsion was obtained fromTianjin chemical reagent Ltd.; YSZ micro/nanoparticle was bought from Cabot Corporation in America, and its average size is $0.08 \sim 30 \mu \mathrm{m}$; PVA was obtained from Beijing Organic Chemistry Company, the degree of polymerization is 1700 , and the degree of alcoholysis is $99 \%$.

\subsection{Membrane preparing}

The specimen compositions were prepared by PTEF resin emulsion, YSZ powders and PVA. First, the mixtures were heated and strived for $30 \sim 60 \mathrm{~min}$ by magnetic stirrer in the range of $60^{\circ} \mathrm{C}$ to $90^{\circ} \mathrm{C}$ which was not expected to be higher than $100^{\circ} \mathrm{C}$ in order to remove water. Second, the mixtures were squeezed and turned out to be the dense and smooth thick film. The membrane with a thickness around $0.2 \sim 0.4 \mathrm{~mm}$ was obtained at room temperature by homemade calendar at a rolling speed between $0.5 \sim 1.5 \mathrm{~m} / \mathrm{min}$, and then it was heated at $50 \sim 100^{\circ} \mathrm{C}$ in the oven. Third, pre-molded YSZ/PTFE composite microporous membrane was prepared with different draw ratio by stretching at a speed of about $0.5 \sim 1 \mathrm{~m} / \mathrm{min}$ and heating at approximately $200^{\circ} \mathrm{C}$. At last, under a certain tension, the pre-molded membrane was heat-setting by calcinations in the range of $300^{\circ} \mathrm{C}$ to $330^{\circ} \mathrm{C}$ for $8 \sim 10 \mathrm{~min}$, and then YSZ/PTFE composite microporous membrane was prepared after natural cooling.

\subsection{Studies of DSC curves}

DSC curves were produced by a differential scanning calorimetry (DSC-7, Perkin-Elmer Limited, American). The samples were analysed under the $\mathrm{N}_{2}$ gas atomsphere at the heating rate of $2^{\circ} \mathrm{C} / \mathrm{min}$ between $40^{\circ} \mathrm{C}$ and $400^{\circ} \mathrm{C}$.

\subsection{Porosity analysis}

Porosity is defined as the percentage of air in the composite membraner. In this study the Saturation weighing method was used to measure the porosity of composite microporous membrane. Ethanol was chosen as the saturated medium in this paper.

\section{Results and discussion}

\subsection{Effect of dispersant on the porosity}

The C-F and C-C in the PTFE molecule are hydrophobic groups, so PTFE is incompatible with YSZ powders. Since that the PVA was used as dispersant. PVA was dissolved in hot water at $90^{\circ} \mathrm{C}$, and the $2 \% \mathrm{PVA}$ solution was obtained, and then YSZ powders were added and ultrasonic cleaner was used to mix well for 30min. Finally, PTFE resin emulsion was poured into the solution which was constantly stired by magnetic stirrer. Composite microporous membrane sample was obtained through the steps in 2.2. The effect of dispersant on the porosity of composite microporous membrane was shown in Table 1. The experiment was carried on the conditions: YSZ content $7 \%$, calcination temperature $320^{\circ} \mathrm{C}$, calcination time $8 \mathrm{~min}$ and heating rate $2{ }^{\circ} \mathrm{C} / \mathrm{min}$

Table 1 shows that the porosity of composite microporous membrane increased significantly at different draw ratio from 0.5 times to 1 times to 1.5 times when the PVA was added as dispersant, as a result of that condensation reaction was occured between the hydroxyl groups of PVA molecule and a amount of the hydroxyl groups on the surface of YSZ. At the same time, long carbon chains of the membrane combined with the PTFE substrates, by which not only the interface state between inorganics and organics were effectively modified, but also the dispersion and anti-sedimentation of the YSZ particles in PTFE resin emulsion were enhanced, so that the membrane were stretched more easily. Consequently, the prorosity of the membran added PVA was higher.

\subsection{The effect of the draw ratio on the porosity}

Figure 1 shows the effect of draw ratio on the porosity of composite microporous membrane. The experiment was carried on the conditions: calcination temperature $320^{\circ} \mathrm{C}$, calcination time $8 \mathrm{~min}$, and heating rate $2^{\circ} \mathrm{C} / \mathrm{min}$

It is evident in Figure 1 that the porosity of the membrane is improved with the draw ratio increasing. The porosity of the membrane which is not stretched is zero, and reaches $73.09 \%$ when the draw ratio 3.5 times and YSZ content $8 \%$ are chosen. In addition, pure PTFE membrane was difficult to stretch in the preparation process, and it was likely that the sample fracture would occur when the draw ratio was more than 2 times. From microstructure analysis, the banded structures of PTFE resin are pulled out from the spherical particles when shear stress is applied to them. By increasing draw ratio, the shear force also increases gradually, which promotes more and more band structure undrew and leads to the micro-fiber length increasing. Meanwhile, the average pore size of specimens also increases, so that the porosity increases. (Hao, 2005, pp. 26-29). 


\subsection{The effect of YSZ content on the porosity}

The effect of YSZ content on the porosity of composite microporous membrane is shown in Figure 2. The experiment was carried on the conditions: calcination temperature $320^{\circ} \mathrm{C}$, calcination time $8 \mathrm{~min}$ and heating rate $2^{\circ} \mathrm{C} / \mathrm{min}$.

The porosity gradually increases with the increase of YSZ content. The explanation over this phenomenon is that the addition of YSZ decreases the bulk desity of PTFE resin baseband, and then reduces the fusion degrees among the resin particles and intermolecular force. So under the same stress, the more YSZ content is, the easyer the microporous can be drawed out, the higher the porosity.

\subsection{The effect of Calcination temperature on the porosity}

The calcination temperature is an important part to the preparation of composite microporous membrane. In order to determine the calcination temperature, the experiment carried out the DSC analysis of PTFE resin in the range from $25^{\circ} \mathrm{C}$ to $400^{\circ} \mathrm{C}$. The DSC curve is shown in Figure 3 .

From Figure 3, it can be seen that the melting temperature of used PTFE resin is about $329^{\circ} \mathrm{C}$. In this paper, YSZ/PTFE composite microporous membrane was prepared, using PTFE resin emulsion as a material. The thickness of sample was $0.2 \sim 1 \mathrm{~mm}$. Early results showed that when the calcination temperature was more than $329^{\circ} \mathrm{C}$, under a certain tension, the sample easily occured to the rupture because the sample was comparative thin. But when the calcination temperature is less than $300^{\circ} \mathrm{C}$, crystallization rate is lower and crystal structure is imperfect, following that unstable size and insufficient strength of the sample. Therefore, the calcination temperature should be chosed in the range of $300^{\circ} \mathrm{C}$ to $330^{\circ} \mathrm{C}$.

Figure 4 shows the effect of calcination temperature on the porosity of composite microporous membrane. The experiment was carried on the conditions: the draw ratio 1.5 times, calcination time $8 \mathrm{~min}$ and heating rate $2^{\circ} \mathrm{C} / \mathrm{min}$.

From Figure 4, it can be seen that as the calcination temperature going up within $300 \sim 320^{\circ} \mathrm{C}$, the porosity of composite microporous membrane gradually increases firstly, and reaches the maximum value at $320^{\circ} \mathrm{C}$, then decreases. The reason is that the length of composite membrane sample is fixed, leading to a certain tensile force generating. With the crystal area gradually melting, the amorphous areas become larger, and then more and more macromolecular chains move toward the direction of internal stress eliminated, thus the micro-fibers shrinks. At the same time, the nodes connected with the micro-fibers are gradually undrawed by the tensile force. The bigger nodes decrease, and the small nodes tend to disappear, following that node size decreases and micro-fiber area increases, so the porosity increases. But when the temperature exceeds $320^{\circ} \mathrm{C}$, the crystal areas tend to melt, leading to the macromolecular chain mobility increasing. The micro-fibers fuse with each other or ruptured by the large internal stress, resulting in the decrease of the pore size and the membrane porosity.

\section{Conclusions}

The effects of the dispersant PVA, the draw ratio, YSZ content and calcination temperature on the porosity of composite microporous membrane were systematically investigated using weight method. The main results are summarized as follows:

1) In this paper, YSZ/PTFE composite microporous membrane was prepared by mechanical tensile method, using PTFE resin emulsion as material and YSZ micro/nanoparticles as reinforcer. This method is simple, low cost and so on, having important theoretical significance and practical value.

2) The study analysed the effect of dispersant PVA on the porosity of composite microporous membrane. The result showes that the porosity of composite microporous membrane with dispersant PVA increaseses significantly.

3) The effects of technological sonditions on the porosity of composite microporous membrane were systematically studied. It is indicated that the porosity increases with the increase of YSZ content, but the composite microporous membrane become difficult to stretch when the YSZ content is over $20 \%$; the porosity increases at first and then decreases with the calcination temperature going up, reaching its maximum value at $320^{\circ} \mathrm{C}$; the porosities for the membranes doped with different YSZ contents are positive proportional to the draw ratio, and it reaches $73.09 \%$ with the draw ratio 3.5 times and YSZ content $8 \%$.

\section{References}

Bao, Yilu, Li, He, \& Zhang Liye. (2006). Preparation of antimicrobial biaxially oriented poly(terafluoroethylene) film. New Chemical Materials, 34(1), 8-11 
Bao, Yilu. (2007). Research and Preparation of antimicrobial biaxially oriented poly(terafluoroethylene) film. Beijing Chemical University

Chen, Shanmei, \& Li, Aoqi. (2003). Two-way stretch PTFE microporous membrane and pore properties, Membrane Science And Technology, 23(2), 20-22

Fang, Zhengping, Cha, Linlin, \& Hu, Qiaoling. (2001). Research Progress in Blending Modification of Polytetrafluoroethylene on Other Engineering Plastics. China Plastica Industry, 15(8), 13-16

Huai, Yongjian, Gao, Jiandong, \& Deng Zhenghua. (2010). Preparation and characterization of a special structural poly(acrylonitrile)-based microporous membrane for lithium-ion batteries. Ionics

Hung, Ming, Hung, DeTsai, \& Fang, Kuan-Zong. (2006). Synthesis and characterization of highly ordered mesoporous YSZ by tri-block copolymer. J Porous Mater, 13, 225-230

Hao, XinMin, Zhang, JianChun, \& Guo, YuHai. (2005). Effect of stretching process on expanded PTFE membrane porous structure. Membrane Scienxe and Technology, 25(4), 26-29

Liu C Z, Wu J Q, \& Ren Q L. (2004). Comparative study on the effect of RF and DBD plasma treatment on PTFE surface modification. Materials Chemistry and Physics, 85(2-3), 340-346

Murali K P, Rajesh S, \& Prakash OM. (2009). Comparison of alumina and magnesia filled PTFE composites for microwave substrate applications. Materials Chemistry and Physics, 113(1), 290-295

Kim J H, Kawai M, \& Yonezawa S. (2008). Improved thermal stability of crosslinked PTFE using fluorine gas treatment. Journal of Fluorine Chemistry, 129(7), 654-657

Sun, Xiaoqing, Luo, Huang, \& Huang, Ping. (2009). Properties of PTFE composite membrane by two-way stretch, Plastic, 38(2), 75-77

Wang, Liangliang, \& Tao, Guoliang. (2004). Research on PTFE Matrix Compsoite with High Thermal Conductivity. China PLastics, 18(4), 26-28

Wei, Qi, Li, Jianlin, \& Song Chunlin. (2005). Preparation and Gas Permeation of Supported $\gamma$-A12O3 Membranes Used as Substrate Layer for Microporous Membranes.Journal of Wuhan University of Technology, 20(3), 27-30

Xie, Gang, \& Huang, Chengya. (2007). Research on Modification Technology of Polytetrafluoroethylene. Synthetic Materials Aging and Application, 36(1), 32-39

Xi Z Y, Xu YY, \& Zhu L P. (2008). Studies on surface grafting of AAc/SSS binary monomers onto polytetrafluoroethylene by dielectric barrier discharge initiation. Applied Surface Science, 254(22), 7469-7476

Yuan, Haigen, Ceng, Jinfang, \& Yang Jie. (2005). Progress in Research on Modification of PTFE. China Plastica Industry, 33(8), 7-11

Table 1. Effect of dispersant on the porosity of composite microporous membrane

\begin{tabular}{|c|c|c|}
\hline draw ratio(times) & without dispersant(\%) & with dispersant PVA(\%) \\
\hline 0.5 & 11.74 & 15.21 \\
\hline 1 & 19.90 & 28.44 \\
\hline 1.5 & 35.55 & 46.77 \\
\hline
\end{tabular}




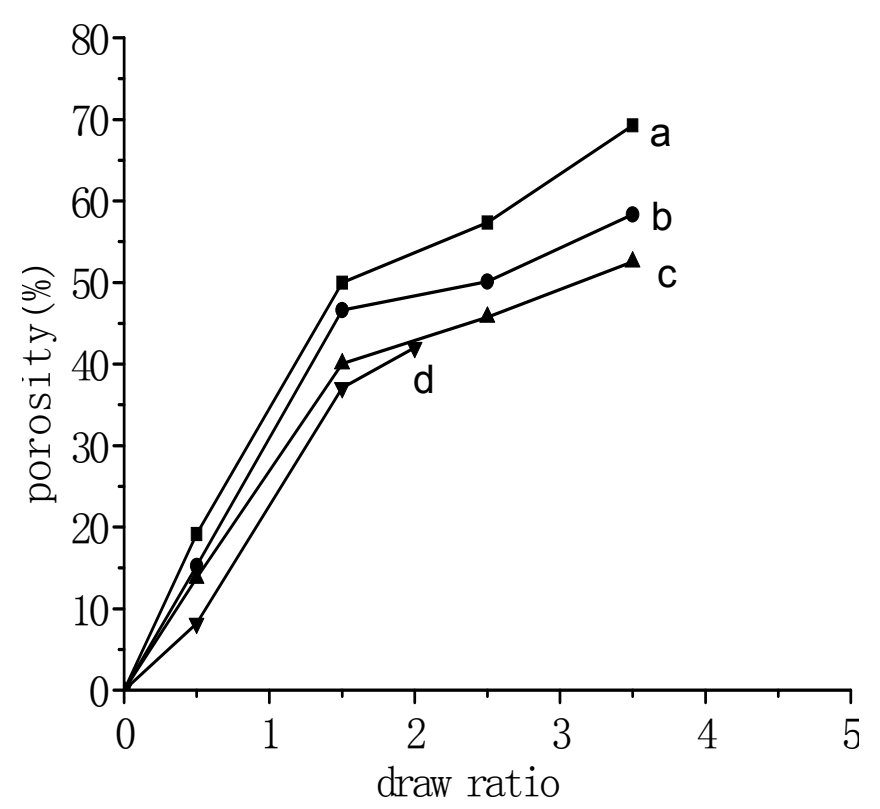

Figure 1. Effect of the draw ratio on the porosity of composite microporous membrane: YSZ content (a) $8 \%$, (b) 7\%, (c) 5\% and (d) pure PTFE membrane

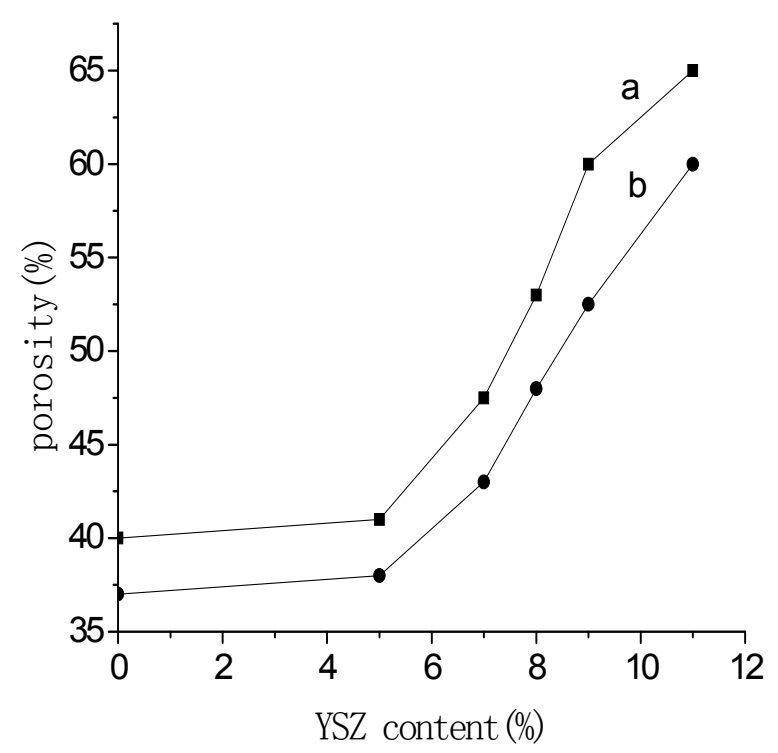

Figure 2. Effect of YSZ content on the microporous of composite microporous membrane:

The draw ratio (a) 2 times and (b) 1.5 times 


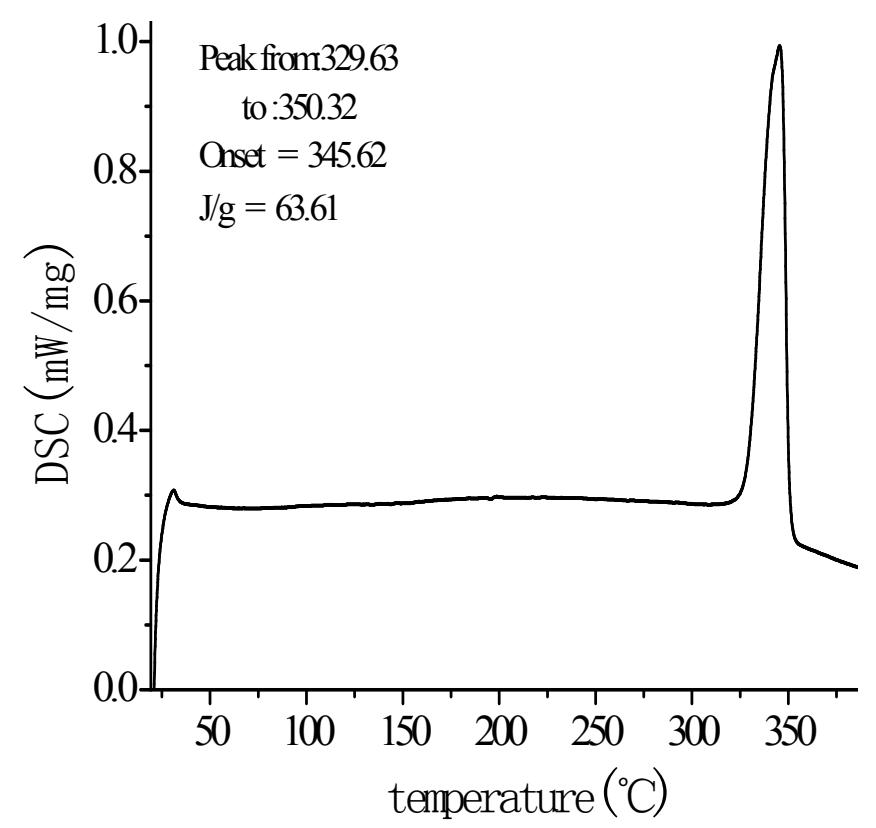

Figure 3. DSC curves of PTFE membrane

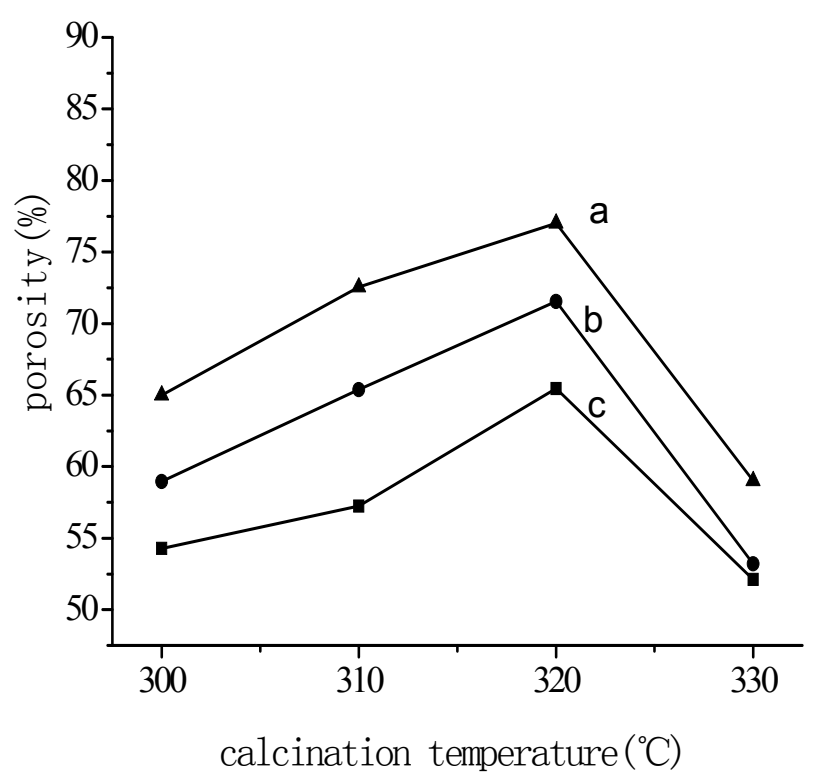

Figure 4. Effect of calcination temperature on the microporous of composite microporous membrane: YSZ content (a) $10 \%$, (b) $7 \%$ and (c) $5 \%$ 